Document downloaded from:

http://hdl.handle.net/10251/51451

This paper must be cited as:

De Pereda Sebastián, D.; Romero Vivó, S.; Ricarte Benedito, B.; Bondía Company, J. (2012). On the prediction of glucose concentration under intra-patient variability in type 1 diabetes: A monotone systems approach. Computer Methods and Programs in Biomedicine. 108(3):993-1001. doi:10.1016/j.cmpb.2012.05.012.

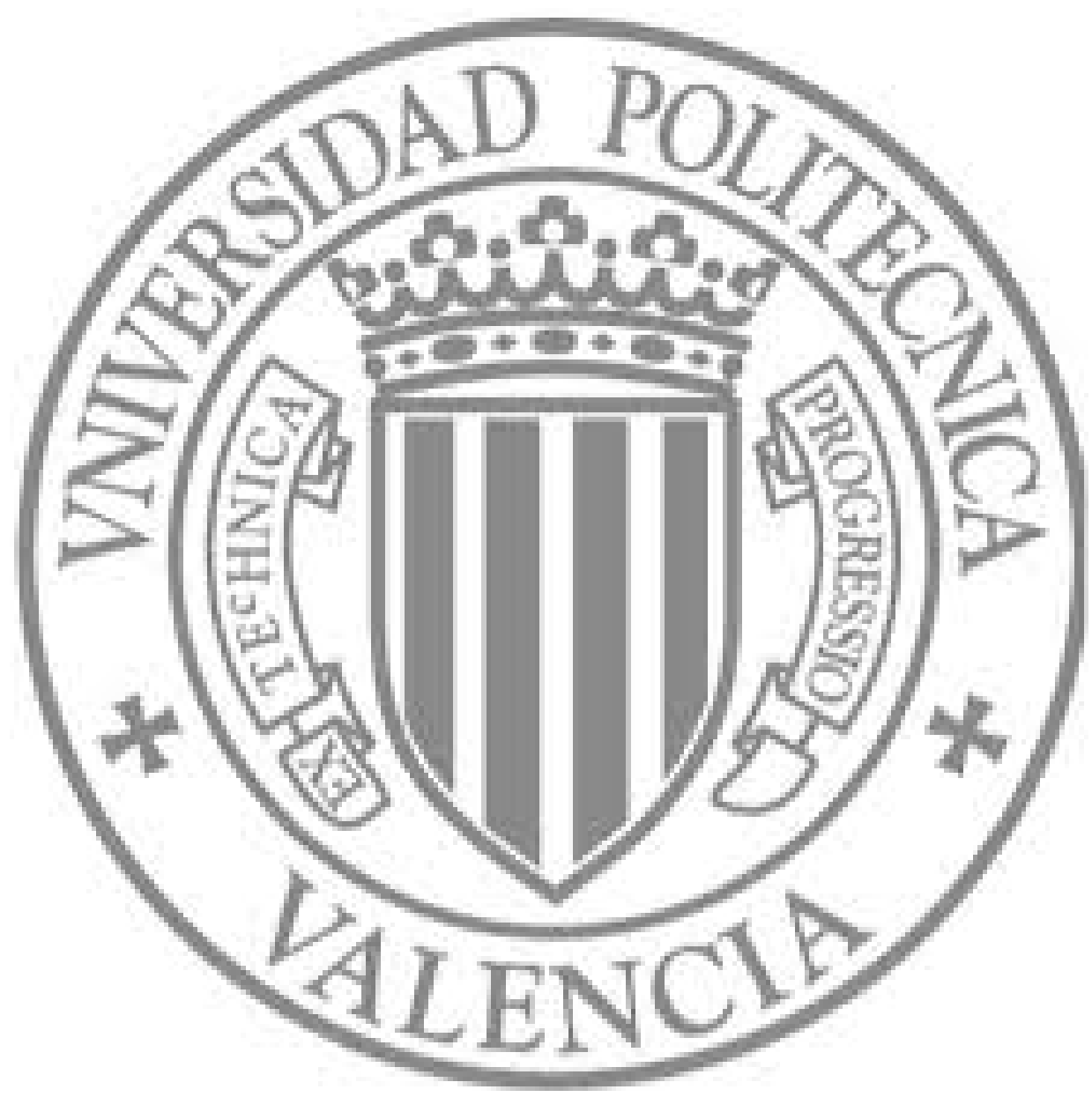

The final publication is available at

http://dx.doi.org/10.1016/j.cmpb.2012.05.012

Copyright Elsevier 


\title{
On the prediction of glucose concentration under intra-patient variability in type 1 diabetes: a monotone systems approach
}

\author{
Diego de Pereda ${ }^{\mathrm{a}}$, Sergio Romero-Vivo ${ }^{\mathrm{b}}$, Beatriz Ricarte ${ }^{\mathrm{b}}$, Jorge Bondia $\mathrm{B}^{\mathrm{a}, *}$ \\ ${ }^{a}$ Institut Universitari d'Automàtica i Informàtica Industrial, Universitat Politècnica de València, Spain \\ ${ }^{b}$ Institut Universitari de Matemàtica Multidisciplinar, Universitat Politècnica de València, Spain
}

\begin{abstract}
Insulin therapy in type 1 diabetes aims to mimic the pattern of endogenous insulin secretion found in healthy subjects. Glucose-insulin models are widely used in the development of new predictive control strategies in order to maintain the plasma glucose concentration within a narrow range, avoiding the risks of high or low levels of glucose in the blood. However, due to the high variability of this biological process, the exact values of the model parameters are unknown, but they can be bounded by intervals. In this work, the computation of tight glucose concentration bounds under parametric uncertainty for the development of robust prediction tools is addressed.

A monotonicity analysis of the model states and parameters is performed. An analysis of critical points, state transformations and application of differential inequalities are proposed to deal with non-monotone parameters. In contrast to current methods, the guaranteed simulations for the glucose-insulin model are carried out by considering uncertainty in all the parameters and initial conditions. Furthermore, no time-discretisation is required, which helps to reduce the computational time significantly. As a result, we are able to compute a tight glucose envelope that bounds all the possible patient's glycemic responses with low computational effort.
\end{abstract}

Keywords: Compartmental models, Interval simulation, Glucose-insulin models, Type 1 diabetes, Blood glucose prediction

\section{Introduction}

Insulin is a hormone secreted by the pancreas with the role of reducing glucose concentration in the blood. Under normal circumstances, insulin secretion maintains the plasma glucose concentration within a narrow range. A decrease in the plasma glucose concentration is followed by a fall in the insulin secretion and an increase of the counter-regulatory hormones like glucagon. On the other hand, the insulin secretion increases when the plasma glucose concentration rises, for instance after a meal intake.

Patients affected by Type 1 Diabetes Mellitus suffer an autoimmune disease characterised by an absolute insulin deficit. When this is untreated, a high level of plasma glucose concentration is obtained (hyperglycemia) leading to deleterious long-term complications. Indeed, exogenous insulin administration is

\footnotetext{
${ }^{*}$ Corresponding author. Tel.: +34 963877007; Fax: +34 963879579

Email address: jbondia@isa.upv.es (Jorge Bondia)

necessary to maintain the plasma glucose concentration within a narrow range (approximately $65-140 \mathrm{mg} / \mathrm{dl}$ ). This is currently done through insulin pens (Multiple Daily Injections) or insulin pumps (Continuous Subcutaneous Insulin Infusion). However, if the insulin administered is more than required, this may lead to a low level of plasma glucose concentration (hypoglycemia), causing unconsciousness and even death. In the last decade, research has been focused on the development of an Artificial Pancreas, i.e., a closed-loop glucose control system that automatically dispenses insulin subcutaneously. In either case, insulin therapy aims to mimic the pattern of the endogenous insulin secretion found in healthy subjects.

A common characteristic of biological processes is variability, leading to large intra-patient and interpatient uncertainty. This variability arises, among other causes, from the different physiological processes involved such as subcutaneous insulin absorption, circadian rhythms of insulin sensitivity, action of counterregulatory hormones, etc. In addition, meal ingestion introduces important disturbances in glucose homeosta- 
sis depending, among other things, on the meal composition and even previous meals. Patient variability can thus be a limiting factor to the performance of openloop (insulin pens and pumps) and closed-loop (artificial pancreas) insulin therapies. Tools are required for the design of robust strategies integrating patient's variability.

Glucose-insulin models are widely used in the development of new predictive control strategies. When intra-patient variability is considered, the exact values of the model parameters are unknown. However, they can be bounded by intervals characterising the observed variability. Efficient methods for the prediction of glycemic responses under intra-patient variability may foster the development of new control strategies such as robust Model Predictive Control [1], robust fault detection [2], robust parameter identification [3], robust hypoglycemia prediction, robust insulin dosage optimisation [4] and robust model-based insulin pump therapies [5].

The aim of this work is to compute tight solution bounds for glucose-insulin models to predict the glucose concentration under intra-patient variability in subjects with type 1 diabetes. Monte Carlo methods have been traditionally used to deal with uncertainty. However, they have not been considered as an effective approach to compute a glucose envelope, as they do not guarantee the inclusion of all the possible glucose values. Independently to the number of simulations performed, there is no guarantee that the output space has been completely covered. Moreover, Monte Carlo techniques generally have a high computational cost, as a high number of simulations is needed to cover the uncertain input space sufficiently.

A preceding contribution by Calm et al. [6] has applied modal interval analysis [7] to compute tight envelopes of glucose concentration under parametric uncertainty. Compared with Monte Carlo simulations, modal interval analysis guarantees that the actual response is inside the computed envelope, and requires much less computational time. However, only some specific parameters of the glucose-insulin model are considered uncertain. Furthermore, Euler timediscretisation of equations is necessary, which may not be efficient from a numerical point of view.

In this work a monotone systems approach for the computation of glucose envelopes is presented. A monotonicity analysis of the model states and parameters is performed. An analysis of critical points, state transformations and application of differential inequalities are proposed to deal with non-monotone parameters. The glucose-insulin model developed by Hov- orka et al. [8], one of the relevant models in literature, has been chosen to illustrate the technique, although the method could be extended to other models. Parametric uncertainty is considered in all the parameters and initial conditions of the model. Additionally, no time-discretisation is required, which helps to reduce the computational time.

The paper has been organised as follows. In Section 2 , interval simulation for an initial value problem for parametric ordinary differential equations (ODEs) is introduced, and the two main approaches are listed. In Section 3, the glucose-insulin model developed by Hovorka et al. [8] is described. In Section 4, the monotonicity of the glucose-insulin equations are analysed with respect to the states and parameters of the model in order to compute the solution envelope under parametric uncertainty. In Section 5, glucose concentration envelopes are computed and compared with numerical simulations. Finally, Section 6 outlines the conclusions of this study.

\section{Initial Value Problems for Parametric ODEs}

Continuous-time compartmental systems under parametric uncertainty are considered. The parameters and initial conditions of the model are unknown, but they can be bounded by intervals. Systems are described by an initial-value problem (IVP):

$$
\begin{aligned}
& \dot{x}(t, p)=f(x, p), \quad x\left(t_{0}\right)=x_{0}, \\
& x \in \mathbb{R}^{n}, t \in \mathbb{R}, p \in \mathbb{R}^{n_{p}}
\end{aligned}
$$

where $x$ is the state vector, $p$ is the parameter vector, $n_{p}$ is the number of parameters, and $x\left(t ; t_{0}, x_{0}, p\right)$ is the solution of (1). Representing intervals in bold, interval vectors $\mathbf{p}$ and $\mathbf{x}_{\mathbf{0}}$ include all the possible values for the parameters $p$ and for the initial conditions $x_{0}$ of the model, respectively. The set of possible solutions considering parametric uncertainty is denoted by $\mathbf{x}\left(t ; t_{0}, \mathbf{x}_{\mathbf{0}}, \mathbf{p}\right)$ :

$$
\mathbf{x}\left(t ; t_{0}, \mathbf{x}_{\mathbf{0}}, \mathbf{p}\right)=\left\{x\left(t ; t_{0}, x_{0}, p\right) \mid x_{0} \in \mathbf{x}_{\mathbf{0}}, p \in \mathbf{p}\right\}
$$

The computation of solution envelopes plays a key role in the simulation of systems under parametric uncertainty. Such a computation can be performed by using one-step-ahead iteration based on previous approximations of the reachable set (region-based approaches), or a set of point-wise trajectories generated by the selection of particular values of the parameters $p \in \mathbf{p}$ and 
initial conditions $x_{0} \in \mathbf{x}_{\mathbf{0}}$ by using heuristics or optimisation (trajectory-based approaches) [9]. Algorithms to compute solution envelopes are classified according to the type of approach.

\subsection{Region-based approaches}

Region-based approaches are performed in two phases [10,11], and applying one-step-ahead iteration based on previous approximations of the reachable set. The first step consists in finding an a priori enclosure $\tilde{\mathbf{x}}_{\mathbf{i}}$ for an interval $\left[t_{i}, t_{i+1}\right]$, supposing $\mathbf{x}_{\mathbf{i}}$ has been computed at $t_{i}$ such that

$$
\mathbf{x}\left(t_{i} ; t_{0}, \mathbf{x}_{\mathbf{0}}, \mathbf{p}\right) \subseteq \mathbf{x}_{\mathbf{i}} .
$$

There is a unique solution of the state vector $\mathbf{x}(t)$ for each $x_{i} \in \mathbf{x}_{\mathbf{i}}, t \in\left[t_{i}, t_{i+1}\right]$, such that

$$
\mathbf{x}\left(t ; t_{i}, \mathbf{x}_{\mathbf{i}}, \mathbf{p}\right) \subseteq \tilde{\mathbf{x}}_{\mathbf{i}} \quad \forall t \in\left[t_{i}, t_{i+1}\right] .
$$

The second step consists in an enclosure of the truncation error of the method through $\tilde{\mathbf{x}}_{\mathbf{i}}$, and the computation of a tighter enclosure $\mathbf{x}_{\mathbf{i}+\mathbf{1}}$ at $t_{i+1}$ such that

$$
\mathbf{x}\left(t_{i+1} ; t_{0}, \mathbf{x}_{\mathbf{0}}, \mathbf{p}\right) \subseteq \mathbf{x}_{\mathbf{i}+\mathbf{1}} \subseteq \tilde{\mathbf{x}}_{\mathbf{i}} .
$$

At each iteration, the true solution set is wrapped into a region-based on outer approximations. This produces an overestimation commonly known as the wrapping effect $[9,10]$. Regions must be feasible to be constructed and represented on a computer, or the region representation will produce a significant overestimation. The errors involved can quickly accumulate, and hence, the solution envelope of the interval system explodes. Several methods have been proposed to avoid the wrapping effect, or at least to reduce it, since it was first observed in the early 1960s [12]. These methods include a change of coordinates [12], the use of Taylor models [13], or a QR-factorisation [14] to rotate the state space of the interval system, as well as the use of ellipsoids [15] or zonotopes [16].

\subsection{Trajectory-based approaches}

The rate of change for each compartment of an ODE compartmental model can be expressed as a function of the rest of compartments at that time:

$$
\begin{aligned}
\dot{x}_{1}(t, p) & =f_{1}\left(t, x_{1}(t), x_{2}(t), \ldots, x_{n}(t), p\right) \\
\dot{x}_{2}(t, p) & =f_{2}\left(t, x_{1}(t), x_{2}(t), \ldots, x_{n}(t), p\right) \\
& \vdots \\
\dot{x}_{n}(t, p) & =f_{n}\left(t, x_{1}(t), x_{2}(t), \ldots, x_{n}(t), p\right)
\end{aligned}
$$

simplified by $\frac{d \mathbf{x}}{d t}=\mathbf{f}(\mathbf{x}, \mathbf{p})$, where $\mathbf{f}$ is the vector function with components $f_{i}$, and all compartments $x_{i}$ of the system take arbitrary non-negative values.

This approach consists in performing a monotonicity analysis of the compartments and parameters of the model. Monotone systems have very robust dynamical characteristics, since they respond to perturbations in a predictable way. The interconnection of monotone systems may be studied in an analytical way [11], by considering a flow $\mathbf{x}(t)=\phi\left(\mathbf{x}_{\mathbf{0}}, t\right)$. A system is monotone if $\mathbf{x}_{\mathbf{0}} \leq \mathbf{y}_{\mathbf{0}} \Rightarrow \phi\left(\mathbf{x}_{\mathbf{0}}, t\right) \leq \phi\left(\mathbf{y}_{\mathbf{0}}, t\right)$ for all $t \geq 0$, where $\leq$ is a given relation order. Cooperative systems form a class of monotone dynamical systems [17] in which

$$
\frac{\partial f_{i}}{\partial x_{j}} \geq 0, \quad \text { for all } i \neq j, t \geq 0
$$

Graph theory also allows analysing monotone and cooperative systems by using a species graph [18], in which a node is assigned for each compartment of the model. If the node $x_{i}$ has no direct effect on node $x_{j}$, the partial derivative $\frac{\partial f_{j}}{\partial x_{i}}(x)$ equals zero; thus no edge is drawn from node $x_{i}$ to node $x_{j}$. If the effect of the node $x_{i}$ on node $x_{j}$ is positive, the derivative is strictly positive, an activation arrow $(\rightarrow)$ is drawn. Finally, if the effect is negative, an inhibition line $(-)$ is drawn. However, if the derivative sign changes depending on the particular entries, both an activation arrow and an inhibition line are drawn from node $x_{i}$ to node $x_{j}$.

A spin assignment is an allocation in which each node has a sign, such that nodes connected by an activation arrow $(\rightarrow)$ have the same sign, while nodes connected by an inhibition line $(-)$ have different signs. If at least one consistent assignment exists, the dynamical system is monotone. Furthermore, if all nodes are connected by activation arrows $(\rightarrow)$, the system is cooperative. A similar graph can be performed using the exact solution of the problem, instead of the ODEs system. Figure 1 shows an example of a monotone system and a nonmonotone system.
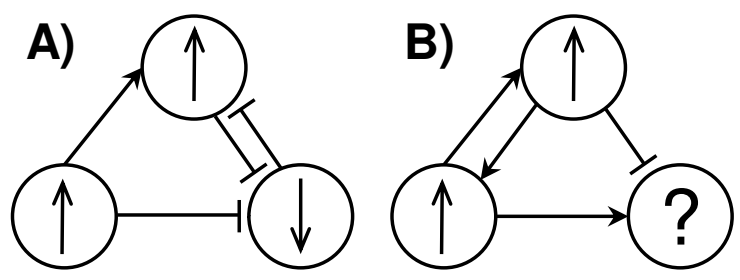

Figure 1: Example of monotone and non-monotone systems. A) A monotone system. B) A non-monotone system. 
In order to calculate solution envelopes, an upper bounding model and a lower bounding model are computed. In an upper bounding model, the cooperative states with respect to the solution take their upper bound value, while the monotone but non-cooperative states, known as competitive states, take the value of their lower bound. On the other hand, a lower bounding model is obtained taking account of the lower bound of the cooperative states, and the upper bound of the competitive states. In both cases, the non-monotone states are still computed as intervals. However, different methods, such as a change of variables or the computation of critical points, can help to reduce the overestimation of the output bounds, as shown in Section 3.

The model parameters are considered as invariant compartments to carry out the monotonicity and cooperativeness analysis, where

$$
\begin{aligned}
\dot{x}_{1}(t) & =f_{1}\left(t, x_{1}(t), x_{2}(t), \ldots, x_{n}(t), p_{1}(t), p_{2}(t), \ldots\right) \\
& \vdots \\
\dot{x}_{n}(t) & =f_{n}\left(t, x_{1}(t), x_{2}(t), \ldots, x_{n}(t), p_{1}(t), p_{2}(t), \ldots\right) \\
\dot{p}_{i}(t) & =0
\end{aligned}
$$

\section{The glucose-insulin model}

The glucose-insulin model is composed of four systems: the carbohydrate digestion and absorption system, the subcutaneous insulin absorption system, the insulin action system, and the glucose metabolism system. Figure 2 shows the input-output relationship among these system parts. All the systems used in this work are introduced in [8], with reported experimental validation results [19].

\subsection{The carbohydrate digestion and absorption system}

This system describes the carbohydrate digestion and its catabolism to glucose. The gut absorption rate $U_{G}(t)$ ( $\mathrm{mmol} / \mathrm{min})$ is given by

$$
U_{G}(t)=\frac{D_{G} A_{G} t e^{-t / t_{\max , G}}}{t_{\max , G}^{2}}
$$

where $D_{G}(\mathrm{mmol})$ is the amount of carbohydrates digested, $A_{G}$ (unitless) is the carbohydrate bioavailability, and $t_{\max , G}(\mathrm{~min})$ is the time-of-maximum appearance of glucose in the accessible glucose compartment.

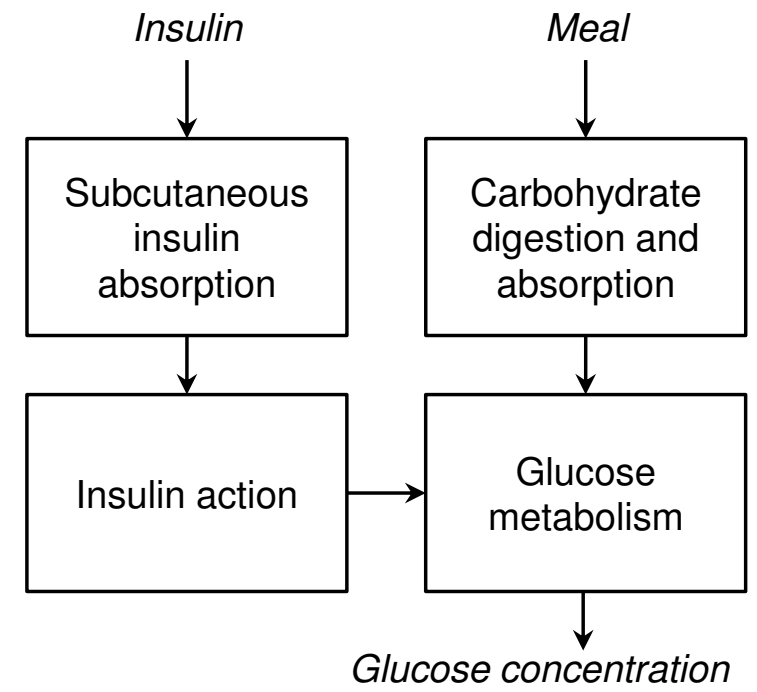

Figure 2: Diagram of the systems interaction.

\subsection{The subcutaneous insulin absorption system}

This system calculates how the administered insulin appears in the blood. It is composed by a twocompartment chain with identical transfer rates $1 / t_{\text {max }, I}$ (min) between the two subcutaneous insulin compartments $S_{1}$ and $S_{2}$ :

$$
\begin{aligned}
& \dot{S}_{1}(t)=u(t)-\frac{S_{1}(t)}{t_{\max , I}} \\
& \dot{S}_{2}(t)=\frac{S_{1}(t)}{t_{\max , I}}-\frac{S_{2}(t)}{t_{\max , I}}
\end{aligned}
$$

where $u(t)(\mathrm{mU} / \mathrm{min})$ represents the administration of insulin (basal and bolus), and $t_{\max , I}(\mathrm{~min})$ is the time-tomaximum insulin absorption. The appearance of insulin in the plasma $U_{I}(t)(\mathrm{mU} / \mathrm{min})$ is given by

$$
U_{I}(t)=\frac{S_{2}(t)}{t_{\max , I}}
$$

The plasma insulin concentration $I(t)(\mathrm{mU} / \mathrm{L})$ is represented by

$$
\dot{I}(t)=\frac{U_{I}(t)}{V_{I}}-k_{e} I(t)
$$

where $V_{I}(\mathrm{~L})$ is the insulin distribution volume, and $k_{e}$ $(1 / \mathrm{min})$ is the fractional elimination rate. 


\subsection{The insulin action system}

The plasma insulin concentration affects the glucose transportation from the plasma to the tissues, the disposal of peripheral glucose, and the production of hepatic glucose. These actions are represented by

$$
\begin{aligned}
& \dot{x}_{1}(t)=-k_{a 1} x_{1}(t)+k_{b 1} I(t) \\
& \dot{x}_{2}(t)=-k_{a 2} x_{2}(t)+k_{b 2} I(t) \\
& \dot{x}_{3}(t)=-k_{a 3} x_{3}(t)+k_{b 3} I(t)
\end{aligned}
$$

where $x_{1}(t)(1 / \mathrm{min})$ represents the effects of insulin on the distribution and transport of glucose, $x_{2}(t)(1 / \mathrm{min})$ stands for the effect on the glucose disposal, while $x_{3}(t)$ (unitless) symbolises the effect on the production of endogenous glucose. The parameters $k_{a i}(1 / \mathrm{min})$, $i=1,2,3$, are the deactivation rate constants, while $k_{b 1}$, $k_{b 2}\left(1 / \mathrm{min}^{2}\right.$ per $\left.\mathrm{mU} / \mathrm{L}\right)$ and $k_{b 3}(1 / \mathrm{min}$ per $\mathrm{mU} / \mathrm{L})$ are the activation rate constants.

\subsection{The glucose metabolism system}

Finally, the glucose kinetics are represented by a twocompartment system, as follows:

$$
\begin{gathered}
\dot{Q}_{1}(t)=-x_{1}(t) Q_{1}(t)+k_{12} Q_{2}(t)-F_{01}^{c}(t) \\
-F_{R}(t)+U_{G}(t)+E G P_{0}\left(1-x_{3}(t)\right) \\
\dot{Q}_{2}(t)=x_{1}(t) Q_{1}(t)-\left(k_{12}+x_{2}(t)\right) Q_{2}(t) \\
G(t)=\frac{Q_{1}(t)}{V_{G}}
\end{gathered}
$$

where $Q_{1}(t)$ and $Q_{2}(t)$ (mmol) are the glucose masses in the accessible compartment and the non-accessible compartment, respectively, while $G(t)(\mathrm{mmol} / \mathrm{L})$ is the glucose concentration in the accessible compartment. The parameter $k_{12}(1 / \mathrm{min})$ is the transfer rate from the non-accessible compartment to the accessible compartment, the parameter $V_{G}(\mathrm{~L})$ denotes the distribution volume of the accessible compartment, and the parameter $E G P_{0}(\mathrm{mmol} / \mathrm{min})$ stands for the endogenous glucose production extrapolated to the zero insulin concentration. The function $F_{01}^{c}(t)(\mathrm{mmol} / \mathrm{min})$ and the parameter $F_{01}$ represent the total non-insulin-dependent glucose disposal, while the function $F_{R}(t)$ is the renal glucose clearance above the glucose threshold of $9 \mathrm{mmol} / \mathrm{L}$ :

$F_{01}^{c}(t)= \begin{cases}\frac{F_{01} G(t)}{4.5} & \text { if } G(t)<4.5 \mathrm{mmol} / \mathrm{L} \\ F_{01} & \text { if } G(t) \geq 4.5 \mathrm{mmol} / \mathrm{L}\end{cases}$

$$
F_{R}(t)= \begin{cases}0 & \text { if } G(t)<9 \mathrm{mmol} / \mathrm{L} \\ 0.003(G(t)-9) V_{G} & \text { if } G(t) \geq 9 \mathrm{mmol} / \mathrm{L}\end{cases}
$$

\section{Solution envelopes under parametric uncertainty}

In this section, the systems presented in the previous section are analysed by trajectory-based approaches, focusing on the parameters monotonicity. Uncertainty is considered for all the parameters, as well as for all the initial conditions of the states.

\subsection{The carbohydrate digestion and absorption system}

The gut absorption rate $U_{G}(t)$ is given by (2), in which the parameters $D_{G}$ and $A_{G}$ are cooperative with respect to $U_{G}(t)$, as seen in Figure 3 . This means that the maximum value of $U_{G}$ is reached only if the maximum values of $D_{G}$ and $A_{G}$ are computed.

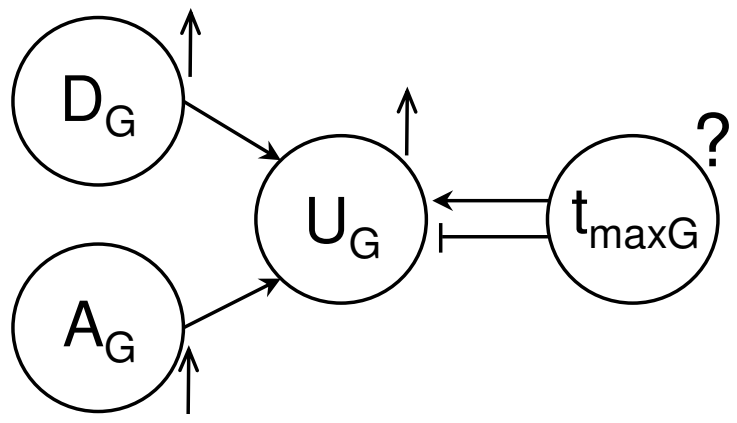

Figure 3: Parameters monotonicity of the carbohydrate digestion and absorption system.

On the other hand, $t_{\max , G}$ is a non-monotone parameter, but the critical points of $U_{G}(t)$ with respect to $t_{\max , G}$ can be obtained for a fixed $t$ :

$$
\frac{\partial U_{G}(t)}{\partial t_{\max , G}}=0 \Rightarrow t_{\max , G}=\frac{t}{2}
$$

The sign of the second derivative of $U_{G}(t)$ with respect to $t_{\max , G}$ determines the stability of the critical point $t / 2$ :

$$
\left.\frac{\partial^{2} U_{G}(t)}{\partial t_{\max , G}^{2}}\right|_{t_{\max , G}=t / 2}=-\frac{32 A_{G} D_{G}}{e^{2} t^{3}}
$$

As the second derivative is negative, $U_{G}(t)$ reaches its maximum value at $t_{\max , G}=t / 2$. However, $t / 2$ is not always a possible value for the interval $t_{\max , G}$, depending on the value of $t$ at each time step. If $t / 2$ is not a possible value for the interval $t_{\max , G}$, the maximum value for 
$U_{G}(t)$ will be attained computing both interval bounds of $t_{\max , G}$ and taking into account the one that maximizes $U_{G}(t)$. On the other hand, the inferior bound of $U_{G}(t)$ is always obtained computing both interval bounds of $t_{\max , G}$ and adopting the one that minimises $U_{G}(t)$.

\subsection{The subcutaneous insulin absorption system}

Insulin can be administered in bolus or basal doses, being cooperative with respect to the model in both cases. Bolus are computed as the initial value of the $S_{1}$ compartment (3), while $u(t)$ denotes the basal doses (3):

$$
S_{1}(0)=u_{\text {bolus }} \quad \text { and } \quad u(t)=u_{\text {basal }}
$$

The problem to solve is a linear ODE system given by (3) and (4), whose solution [20] is given by

$$
\begin{aligned}
& U_{I}(t)=u_{\text {basal }}+\frac{e^{-\left(t / t_{\text {max }, I}\right)} t\left(-t_{\text {max }, I} u_{\text {basal }}+u_{\text {bolus }}\right)}{t_{\text {max }, I}^{2}} \\
& +e^{-\left(t / t_{\text {max }, I}\right)}\left(-u_{\text {basal }}+U_{I}(0)\right)
\end{aligned}
$$

with two critical points with respect to the parameter $t_{\text {max }, I}$. In order to obtain $U_{I}(t)$ envelope, instead of working with $t_{\max , I}$ as an interval, both critical points (if these values are inside the $t_{\max , I}$ interval) and interval bounds are computed.

The plasma insulin concentration $I(t)$, given by (5), is cooperative with respect to $U_{I}(t)$, while the parameters $V_{I}$ and $k_{e}$ are competitive, as seen in Figure 4.

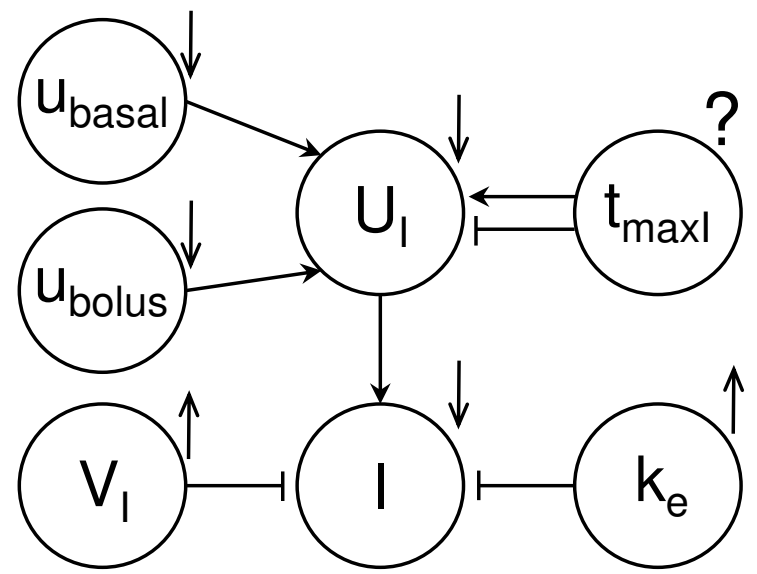

Figure 4: Parameters monotonicity of the subcutaneous insulin absorption system.

\subsection{The insulin action system}

The effects of insulin on the glucose transport from the plasma to the tissues, the disposal of the peripheral glucose, and the production of hepatic glucose are given by (6). Analysing the equations of the system, it is clear that $I(t)$ and $x_{i}, i=1,2,3$ are all cooperative. Furthermore, the parameters $k_{b i}, i=1,2,3$, are also cooperative, while the parameters $k_{a i}, i=1,2,3$, are competitive, as seen in Figure 5.

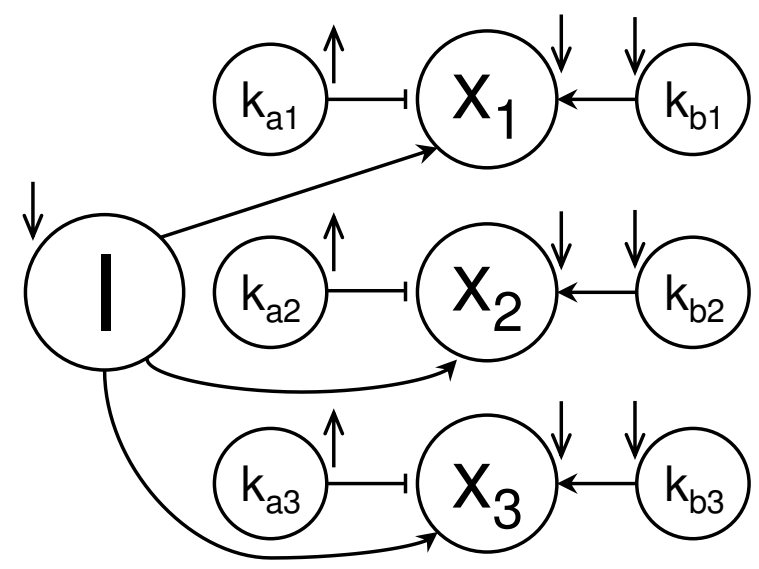

Figure 5: Parameters monotonicity of the insulin action system.

\subsection{The glucose metabolism system}

The equations of the glucose metabolism system (7) are modified to avoid the parameters multi-incidence in different equations. First of all, the non-insulindependent glucose disposal (8) and the renal glucose clearance (9) are transformed such that $F_{01}^{c}(t)+F_{R}(t)=$ $F C(t) G(t)+F R(t)$, as done in [6], where

$$
\begin{gathered}
F C(t)= \begin{cases}\frac{F_{01}}{4.5} & \text { if } G(t)<4.5 \mathrm{mmol} / \mathrm{L} \\
0.003 V_{G} & \text { if } G(t) \geq 9 \mathrm{mmol} / \mathrm{L} \\
0 & \text { otherwise }\end{cases} \\
F R(t)= \begin{cases}0 & \text { if } G(t)<4.5 \mathrm{mmol} / \mathrm{L} \\
F_{01}-0.027 V_{G} & \text { if } G(t) \geq 9 \mathrm{mmol} / \mathrm{L} \\
F_{01} & \text { otherwise }\end{cases}
\end{gathered}
$$


As $G(t)$ is given by $G(t)=Q_{1}(t) / V_{G}$, then $\dot{G}(t)=$ $\dot{Q}_{1}(t) / V_{G}$. Thus, by expression (7):

$$
\begin{aligned}
& \dot{G}(t)=-x_{1}(t) G(t) \\
& +\frac{k_{12} Q_{2}(t)-F C(t) G(t)-F R(t)+U_{G}(t)+E G P_{0}\left(1-x_{3}(t)\right)}{V_{G}}
\end{aligned}
$$$$
\dot{Q}_{2}(t)=x_{1}(t) V_{G} G(t)-\left(k_{12}+x_{2}(t)\right) Q_{2}(t)
$$

To avoid the parameter $V_{G}$ multi-incidence in both equations, a new state $H(t)=Q_{2}(t) / V_{G}$ is defined. Hence:

$$
\begin{aligned}
& \dot{G}(t)=-x_{1}(t) G(t)+k_{12} H(t) \\
& +\frac{-F C(t) G(t)-F R(t)+U_{G}(t)+E G P_{0}\left(1-x_{3}(t)\right)}{V_{G}} \\
& \dot{H}(t)=x_{1}(t) G(t)-\left(k_{12}+x_{2}(t)\right) H(t)
\end{aligned}
$$

Finally, to avoid the multi-incidence of the state $x_{1}(t)$ and the parameter $k_{12}$, another new state $S(t)=H(t)+$ $G(t)$ is computed:

$$
\begin{aligned}
& \dot{G}(t)=-x_{1}(t) G(t)+k_{12}(S(t)-G(t)) \\
& +\frac{-F C(t) G(t)-F R(t)+U_{G}(t)+E G P_{0}\left(1-x_{3}(t)\right)}{V_{G}}
\end{aligned}
$$

$\dot{S}(t)=-x_{2}(t)(S(t)-G(t))$

$$
+\frac{-F C(t) G(t)-F R(t)+U_{G}(t)+E G P_{0}\left(1-x_{3}(t)\right)}{V_{G}}
$$

The condition $\operatorname{sign}(\partial \dot{G}(t) / \partial S(t))=\operatorname{sign}(\partial \dot{S}(t) / \partial G(t))$ is necessary to prove the monotonicity between the states $G(t)$ and $S(t)$. As $\partial \dot{G}(t) / \partial S(t)=k_{12} \geq 0$, both states are cooperative if $\partial \dot{S}(t) / \partial G(t)=x_{2}(t)-F C(t) / V_{G}$ is non-negative.

As this condition is not always true, two models are computed: an upper bounding model and a lower bounding model, in which elimination rates are modified to satisfy $x_{2}(t)-F C(t) / V_{G} \geq 0$. In the lower bounding model, the state $x_{2}(t)$ is replaced by $\max \left(x_{2}(t), F C(t) / V_{G}\right)$, increasing its value. As $x_{2}(t)$ is a competitive state, the model obtained is a lower bounding model [21] of the model (10), and such that

$$
\begin{aligned}
& \dot{G}_{1}(t)=-x_{1}(t) G_{1}(t)+k_{12}\left(S_{1}(t)-G_{1}(t)\right) \\
& +\frac{-F C(t) G_{1}(t)-F R(t)+U_{G}(t)+E G P_{0}\left(1-x_{3}(t)\right)}{V_{G}} \\
& \dot{S}_{1}(t)=-\max \left(x_{2}(t), \frac{F C(t)}{V_{G}}\right)\left(S_{1}(t)-G_{1}(t)\right) \\
& +\frac{-F C(t) G_{1}(t)-F R(t)+U_{G}(t)+E G P_{0}\left(1-x_{3}(t)\right)}{V_{G}}
\end{aligned}
$$

For the upper bounding model, $F C(t) / V_{G}$ is replaced by $\min \left(x_{2}(t), F C(t) / V_{G}\right)$, decreasing its value. As $F C(t) / V_{G}$ is competitive, an upper bounding model of (10) is obtained, such that

$$
\begin{aligned}
\dot{G}_{2}(t) & =-x_{1}(t) G_{2}(t)+k_{12}\left(S_{2}(t)-G_{2}(t)\right) \\
& -\min \left(x_{2}(t), \frac{F C(t)}{V_{G}}\right) G_{2}(t) \\
& +\frac{-F R(t)+U_{G}(t)+E G P_{0}\left(1-x_{3}(t)\right)}{V_{G}} \\
\dot{S}_{2}(t) & =-x_{2}(t)\left(S_{2}(t)-G_{2}(t)\right) \\
& -\min \left(x_{2}(t), \frac{F C(t)}{V_{G}}\right) G_{2}(t) \\
& +\frac{-F R(t)+U_{G}(t)+E G P_{0}\left(1-x_{3}(t)\right)}{V_{G}}
\end{aligned}
$$

where $(11) \leq(10) \leq(12)$, and $G_{i}(t)$ and $S_{i}(t), i=1,2$, are cooperative systems. The system (11) is used to calculate the lower bound of $G(t)$, and the system (12) for the upper bound. In both systems, the states $x_{i}(t)$, $i=1,2,3$, are competitive, while the state $U_{G}(t)$ is cooperative. Furthermore, the parameter $k_{12}$ is cooperative and $F_{01}$ is competitive. Finally, the parameter $E G P_{0}$ is monotone for all instant $t$, but it can be cooperative if $\left(1-x_{3}(t)\right)>0$, or competitive otherwise. Also, the parameter $V_{G}$ is always monotone for all instant $t$, but can be cooperative or not depending on whether it divides a positive or a negative quantity, respectively. Thus both intervals bounds for the parameters $E G P_{0}$ and $V_{G}$ have to be computed depending on the condition, as seen in Figure 6.

\section{Results}

The glucose-insulin model developed by Hovorka et al. [8] has been analysed under parametric uncertainty. The parameter values used in all the systems of the model were taken from [8]. As an illustration of the overestimation made, $5 \%$ uncertainty was considered in all the parameters and initial conditions of the model. The simulations were performed for a patient weight of $80 \mathrm{~kg}$, during the 5 hour period that follows a meal of $60 \mathrm{~g}$ and an insulin dose of $3 \mathrm{U}$, taking into account an insulin basal infusion of $0.32 \mathrm{U} / \mathrm{h}$.

All the simulations have been executed with Matlab software (version R2007b) using an Intel(R) 3.2 GHz Pentium(R) processor. We have computed the solution envelope for two types of situations: long-term predictions during a 5-hour postprandial period, and shortterm predictions in which the glucose concentration 


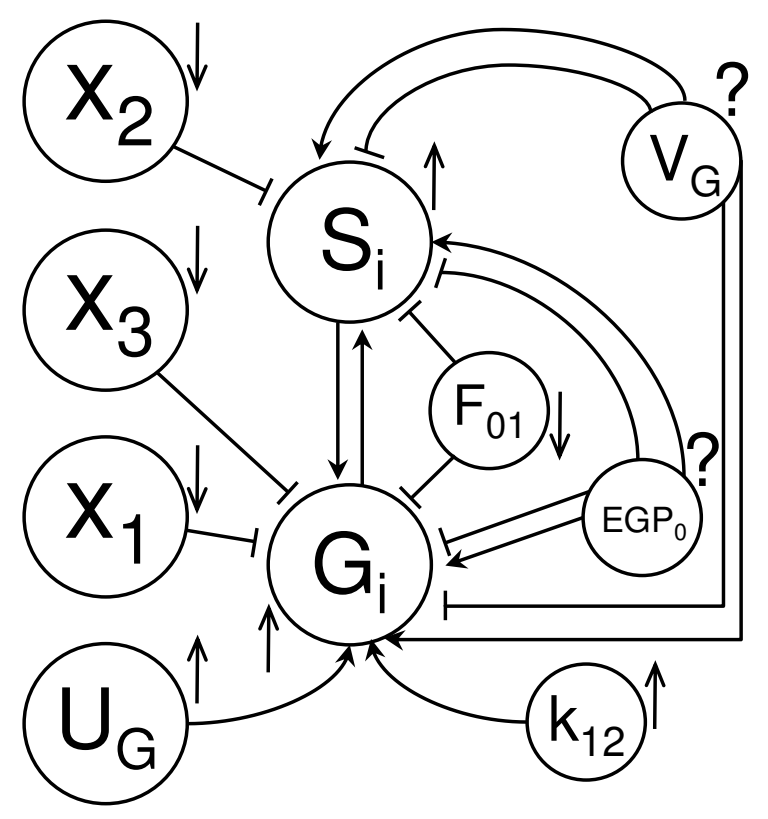

Figure 6: Parameters monotonicity of the glucose metabolism system.

is measured every 15 minutes. Long-term predictions have been used for insulin dose optimisation in Multiple Daily Injections [4] minimising a compound index of hypoglycemia and hyperglycemia risk, and in robust model-based insulin pump therapies using set-inversion techniques [5]. Short-term predictions are used in the context of Model Predictive Control [8] or robust hypoglycemia prediction, where information of new measurements is fed into the predictor.

\subsection{Long-term glucose prediction}

In the long-term prediction examples a unique glucose measurement is performed, at time zero. Different scenarios have been computed by varying the initial blood glucose in each simulation. The resulting solution envelope has been compared with numerical simulations to estimate the overestimation produced. The light grey lines represent several possible numerical simulations performed by varying the parameters and initial conditions values (Monte Carlo approaches), while the black dashed lines represent the computed output bounds.

In the first simulation, a safe initial condition of 150 $\mathrm{mg} / \mathrm{dL}$ was computed, as seen in Figure 7 . In the second scenario, the initial condition was near hypoglycemia with $80 \mathrm{mg} / \mathrm{dL}$, as seen in Figure 8 . Finally, an initial condition near hyperglycemia with $250 \mathrm{mg} / \mathrm{dL}$ was computed, as seen in Figure 9.

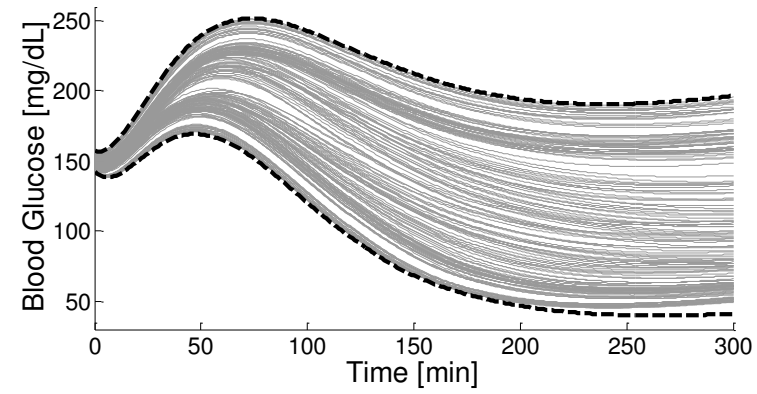

Figure 7: Solution envelope obtained for the first scenario.

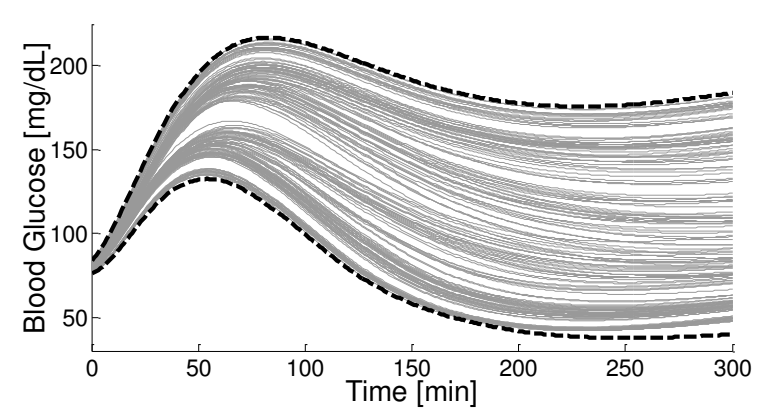

Figure 8: Solution envelope obtained for the second scenario.

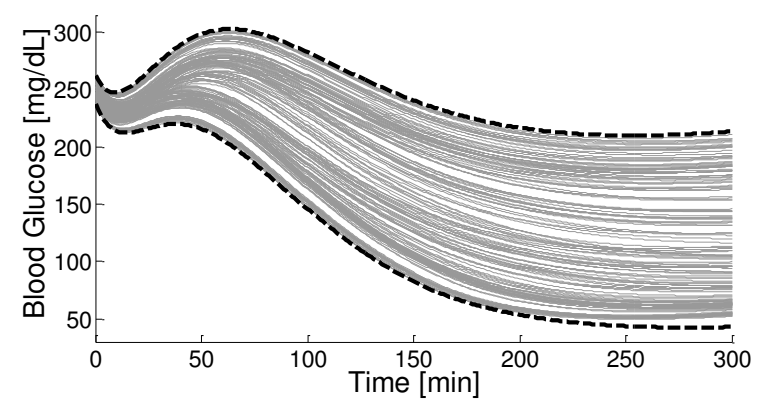

Figure 9: Solution envelope obtained for the third scenario.

\subsection{Short-term glucose prediction}

In the previous example, the glucose level is only measured initially. However, continuous glucose monitoring allows for the frequent measurement of the glucose level in sensor-augmented pumps and the artificial pancreas. In this case, short-term predictions are used at each sample period to predict hypoglycemia or decide the optimal insulin infusion. In this short-term simulation, 30-minutes-ahead predictions are performed every 15 minutes, after each glucose level measurement (generated from a random nominal value for the parameters inside the given intervals). As glucose measurements 
may include an error, 5\% uncertainty is considered in all the glucose values.

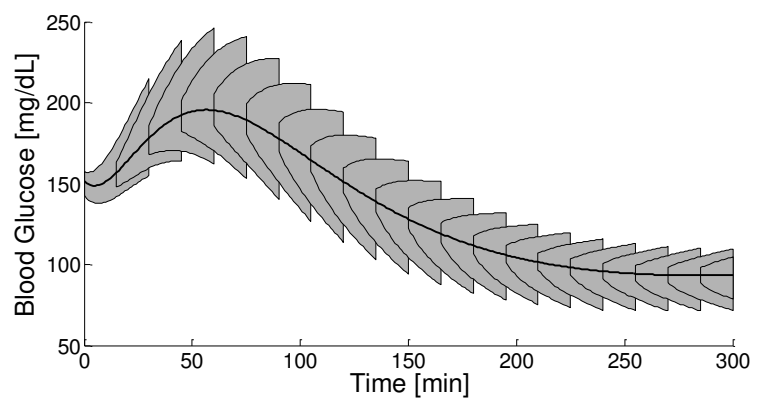

Figure 10: Solution envelope for 30-minute-ahead predictions with glucose measurements every 15 minutes.

\section{Discussion and Conclusion}

The simulations carried out in the previous section are illustrative of the computational needs in a variety of problems where long- or short-term glucose predictions are used. The computation of tight glucose envelopes for long-term predictions is important for the parameter identification [3], to develop new insulin pump therapies [5], and to perform insulin dosage optimisation [4] These tools are fundamental in reducing the risks of hyperglycemic and hypoglycemic episodes, which can be deleterious for patients health. Furthermore, tight glucose envelopes for short-term predictions may produce more robust and safer insulin infusion algorithms, including robust fault detection [2]. Consideration of intra-patient variability into the simulation allows to increase the robustness of these methodologies, yielding to safer systems.

In this work, a monotonicity and critical points analysis of all the model parameters has been proven as a successful tool to compute solution envelopes of the glucose-insulin model considering parametric uncertainty. Nevertheless, as not all the compartments and parameters of the model satisfy the monotonicity conditions, we have considered an upper bounding model and a lower bounding model, which satisfy the monotonicity conditions, to compute a tight glucose envelope for the original glucose-insulin model.

In the case of long-term predictions (Figures 7 to 9), the computed envelope is almost perfect during the first 200 minutes, and then only a small overestimation is made. The total error is smaller than $1.85 \%$, and it is measured by comparing the area of the numerical simulations (Monte Carlo approaches) with the area of the computed solution envelope. The computational cost does not depend on the number of uncertain parameters, and it takes around 0.027 seconds to obtain the solution envelope, using an explicit Runge-Kutta method for the numerical simulation of the bounding systems. It is noteworthy that the consideration of only $5 \%$ uncertainty in all the parameters translates into a wide range of possible patient responses from hypoglycemia to hyperglycemia. Note that this is not due to the simulation overestimation, since the same effect is observed in the Monte Carlo simulations. This fact illustrates the difficulty of glycemic control in clinical practice.

In the case of short-term predictions, the solution envelope includes all the possible responses for the glucose level as expected. As the glucose measurements are performed every 15 minutes, the range of the glucose envelope is much smaller than in the long-term predictions. If the glucose measurements include less uncertainty, or if they are performed with a higher frequency, the range of the glucose envelope will be reduced even more. Observe that the computed glucose range is bigger around the glucose peak value and it reduces as euglycemia is reached again. This is due to the high influence of the uncertainty induced by the meal intake, which vanishes when the equilibrium point is attained. Compared to traditional approaches, a worstcase analysis can be carried out to produce more robust and safer decisions at each sample period.

A preceding contribution by Calm et al. [6] has tackled the same problem. However, some model parameters cannot be considered uncertain, which makes not possible a direct comparison with our method. On the other hand, although Monte Carlo approaches do not guarantee the inclusion of all the possible glucose values, these simulations have been used to estimate an upper bound of the overestimation produced by our proposed method.

Finally, one of the strengths of the method proposed is that, although it has been applied to compute tight solution bounds of the glucose-insulin model developed by Hovorka et al. [8], it can also be applied to other glucose-insulin models, or even for compartmental models that mimic other type of processes. In addition, the method computes guaranteed bounds with few overestimation and low computational cost, which make it suitable for real-time computation. The limitation of this approach is that, as the dynamic model structures differ, each model has to be analyzed independently. Complexity of the analysis of critical points (when required) will also increase with the complexity of the model.

In conclusion, monotone systems approach is an ef- 
ficient tool to compute tight solution envelopes for glucose-insulin models under intra-patient variability. These tools may foster the development of more robust and safe algorithms for glycemic control in type 1 diabetes

\section{Acknowledgements}

This work was partially supported by the Spanish Ministerio de Ciencia e Innovación through Grant DPI-2010-20764-C02, by the Universitat Politècnica de València through Grant PAID-05-09-4334, and by the Generalitat Valenciana through Grant GV/2012/085.

\section{Conflict of interest}

No competing financial interests exist.

\section{References}

[1] J. Bravo, T. Alamo, E. Camacho, Robust mpc of constrained discrete-time nonlinear systems based on approximated reachable sets, Automatica 42 (10) (2006) 1745-1751.

[2] J. Armengol, J. Vehí, M. Sainz, P. Herrero, E. Gelso, Squaltrack: a tool for robust fault detection, Systems, Man, and Cybernetics, Part B: Cybernetics, IEEE Transactions on 39 (2) (2009) 475488.

[3] R. Calm, M. Sainz, P. Herrero, J. Vehi, J. Armengol, Parameter identification with quantifiers, in: Robust Control Design, Vol. 5, 2006, pp. 707-712.

[4] M. García-Jaramillo, R. Calm, J. Bondia, C. Tarín, J. Vehí, Insulin dosage optimization based on prediction of postprandial glucose excursions under uncertain parameters and food intake, Computer Methods and Programs in Biomedicine 105 (1) (2012) $61-69$.

[5] A. Revert, R. Calm, J. Vehi, J. Bondia, Calculation of the best basal-bolus combination for postprandial glucose control in insulin pump therapy, IEEE Transactions on Biomedical Engineering 58 (2) (2011) 274-281.

[6] R. Calm, M. García-Jaramillo, J. Bondia, M. Sainz, J. Vehí, Comparison of interval and Monte Carlo simulation for the prediction of postprandial glucose under uncertainty in type 1 diabetes mellitus, Computer Methods and Programs in Biomedicine 104 (3) (2011) 325 - 332.

[7] E. Gardeñes, M. A. Sainz, L. Jorba, R. Calm, R. Estela, H. Mielgo, A. Trepat, Model intervals, Reliable Computing 7 (2001) 77-111.

[8] R. Hovorka, V. Canonico, L. Chassin, U. Haueter, M. MassiBenedetti, M. Federici, T. Pieber, H. Schaller, L. Schaupp, T. Vering, et al., Nonlinear model predictive control of glucose concentration in subjects with type 1 diabetes, Physiological measurement 25 (2004) 905.

[9] V. Puig, A. Stancu, J. Quevedo, Simulation of uncertain dynamic systems described by interval models: A survey, in: 16th IFAC World Congress, 2005.

[10] N. Nedialkov, Interval tools for ODEs and DAEs, in: SCAN 2006: 12th GAMM-IMACS International Symposium on Scientific Computing, Computer Arithmetic and Validated Numerics, IEEE, 2006, pp. 4-4.
[11] M. Kieffer, E. Walter, Guaranteed nonlinear state estimator for cooperative systems, Numerical Algorithms 37 (1) (2004) 187198.

[12] R. Moore, Interval analysis, Vol. 60, Prentice-Hall Englewood Cliffs, 1966.

[13] K. Makino, M. Berz, Rigorous integration of flows and odes using taylor models, in: Proceedings of the 2009 conference on Symbolic numeric computation, ACM, 2009, pp. 79-84.

[14] R. Lohner, Enclosing the solutions of ordinary initial and boundary value problems, Computer Arithmetic: Scientific Computation and Programming Languages (1987) 255-286.

[15] A. Neumaier, The wrapping effect, ellipsoid arithmetic, stability and confidence regions, Computing. Supplementum 9 (1993) 175-190.

[16] W. Kuhn, Rigorously computed orbits of dynamical systems without the wrapping effect, Computing 61 (1) (1998) 47-67.

[17] H. Smith, Monotone dynamical systems: An introduction to the theory of competitive and cooperative systems, AMS Bookstore, 2008.

[18] E. Sontag, Monotone and near-monotone biochemical networks, Systems and Synthetic Biology 1 (2) (2007) 59-87.

[19] R. Hovorka, F. Shojaee-Moradie, P. Carroll, L. Chassin, I. Gowrie, N. Jackson, R. Tudor, A. Umpleby, R. Jones, Partitioning glucose distribution/transport, disposal, and endogenous production during ivgtt, American Journal of PhysiologyEndocrinology And Metabolism 282 (5) (2002) E992.

[20] D. de Pereda, S. Romero-Vivo, J. Bondia, On the computation of output bounds on parallel inputs pharmacokinetic models with parametric uncertainty, Mathematical and Computer Modellingdoi:10.1016/j.mcm.2011.11.031.

[21] K. Bondar, Some scalar difference inequalities, Applied Mathematical Sciences 5 (60) (2011) 2951-2956. 